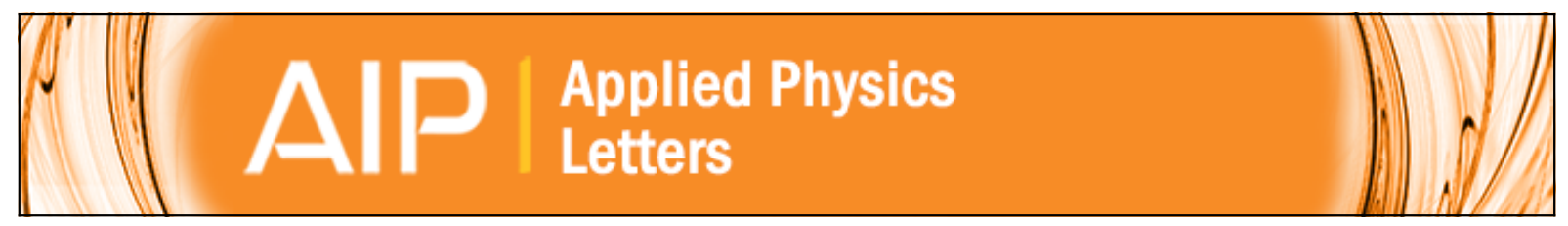

\title{
Carbon nanotube diode fabricated by contact engineering with self-assembled molecules
}

Byoung-Kye Kim, Ju-Jin Kim, Hye-Mi So, Ki-jeong Kong, Hyunju Chang, Jeong-O Lee, and Noejung Park

Citation: Applied Physics Letters 89, 243115 (2006); doi: 10.1063/1.2403929

View online: http://dx.doi.org/10.1063/1.2403929

View Table of Contents: http://scitation.aip.org/content/aip/journal/apl/89/24?ver=pdfcov

Published by the AIP Publishing

\section{Articles you may be interested in}

Electrical transport properties of isolated carbon nanotube/Si heterojunction Schottky diodes

Appl. Phys. Lett. 103, 193111 (2013); 10.1063/1.4829155

An ultra-low leakage current single carbon nanotube diode with split-gate and asymmetric contact geometry

Appl. Phys. Lett. 103, 133508 (2013); 10.1063/1.4823602

Microwave rectification by a carbon nanotube Schottky diode

Appl. Phys. Lett. 93, 043120 (2008); 10.1063/1.2939095

Carbon nanotube Schottky diode and directionally dependent field-effect transistor using asymmetrical contacts Appl. Phys. Lett. 87, 253116 (2005); 10.1063/1.2149991

Modeling of kink-shaped carbon-nanotube Schottky diode with gate bias modulation

Appl. Phys. Lett. 80, 4027 (2002); 10.1063/1.1481213

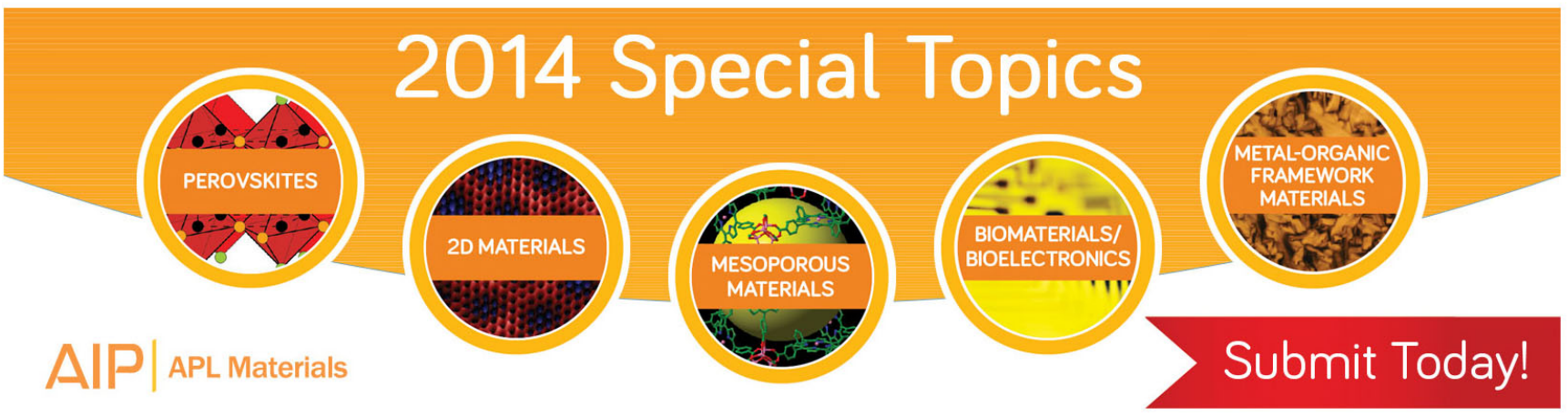




\title{
Carbon nanotube diode fabricated by contact engineering with self-assembled molecules
}

\author{
Byoung-Kye Kim and Ju-Jin Kim \\ Department of Physics, Chonbuk National University, Jeonju 561-756, Korea \\ Hye-Mi So, Ki-jeong Kong, Hyunju Chang, and Jeong-O Lee ${ }^{\text {a) }}$ \\ Advanced Materials Division, Korea Research Institute of Chemical Technology, Daejeon 305-600, Korea \\ Noejung Park \\ Department of Applied Physics, Dankook University, Seoul 140-714, Korea
}

(Received 3 August 2006; accepted 6 November 2006; published online 13 December 2006)

\begin{abstract}
The authors report the construction of carbon nanotube Schottky diodes by covering a selectively exposed area of the electrode with self-assembling molecules. Two self-assembling molecules with different polarities, 2-aminoethanethiol and 3-mercaptopropionic acid, were used to modify the Fermi level lineup at the selected contact. The devices showed $p$-type behavior with symmetric $I-V$ showing clear rectifying behavior after treatment of one contact with 2 -aminoethanethiol. Their experiment, in conjunction with the results of ab initio electronic structure calculations, suggests that the diode action stems from the asymmetric Fermi level lineup between the bare and engineered contacts. (C) 2006 American Institute of Physics. [DOI: 10.1063/1.2403929]
\end{abstract}

The excellent electrical properties of single-walled carbon nanotubes (SWNTs) have generated immense interest from researchers seeking to fabricate nanoscale devices. ${ }^{1} \mathrm{Al}-$ ready, SWNT field effect transistors (FETs) have been developed that outperform silicon devices in some aspects, ${ }^{2-4}$ and research efforts to understand the underlying mechanism and improve the performance of SWNT-FETs are ongoing.

In the present work we focused on the fabrication of a SWNT device that operates as a rectifying diode. Several research groups have fabricated SWNT-based diodes, ${ }^{5-8}$ either using conventional approaches such as selective doping in part of the nanotube $e^{5,6}$ or by adopting local split gates to design the carrier concentration in the nanotube. ${ }^{7,8}$ However, by exploiting the well-known characteristics of the Schottky barrier of SWNT-FET, it may be possible to construct a SWNT-based diode simply by adjusting the Fermi level lineup at the nanotube-metal contact.

The Schottky barrier at the metal-nanotube contact has been noted as a key parameter determining SWNT-FET performance. ${ }^{9,10}$ Common $p$-type operations are largely due to a low Schottky barrier for hole transport at the metalnanotube contact. In this respect, the fabrications of $n$-type SWNT-FETs have proved challenging and interesting. Nosho et al. and $\mathrm{Oh}$ et al. constructed a $n$-type FET by using a low work function metal electrode. ${ }^{11,12}$ Researchers have also tried to construct an asymmetric contact electrode to achieve Schottky diode operation in SWNT-FETs. ${ }^{13-15}$

In the present work, we fabricated symmetric $p$-type SWNT-FETs with high work function metals, and then converted these devices into Schottky diodes by modifying one of the contact barriers with a self-assembled monolayer (SAM) of molecules containing a thiol group.

The SWNT-FETs used in this study were fabricated by the patterned growth method, ${ }^{16}$ and conventional photolithography followed by lift-off. Figure 1(a) shows an atomic force microscope (AFM) image of the bare device used in

\footnotetext{
${ }^{a)}$ Electronic mail: jolee@krict.re.kr
}

our experiments (i.e., before SAM treatment). Onto this bare device, patterns were made with SU-8 negative photoresist to expose one of the contacts for chemical treatment, as depicted in Fig. 1(b). Two self-assembling molecules, 2-aminoethanethiol $\left[\mathrm{HSCH}_{2} \mathrm{CH}_{2} \mathrm{NH}\right.$; molecular weight $(\mathrm{Mw})$ : 77.15] and 3-mercaptopropionic acid $\left(\mathrm{HSCH}_{2} \mathrm{CH}_{2} \mathrm{CO}_{2} \mathrm{H} ; \mathrm{Mw}\right.$ : 106.14), were purchased from Sigma-Aldrich, and used without further treatment. These molecules were selected because they each contain a thiol group, which is well known to favor the formation of highly ordered monolayers on Au surfaces. ${ }^{17}$ Figure 1(c) shows the molecular structures of 2-aminoethanethiol and 3-mercaptopropionic acid, and the predicted directions of the dipole moments of the pristine molecules.

The electrical transport characteristics of the devices were recorded first without molecular treatment, and then the device was reacted with a $10 \mathrm{mM}$ solution of self-assembling molecules in ethanol. Figure 2 shows the $I-V$ characteristics of the SWNT-FET before (black curve) and after treatment of the exposed contact with 2-aminoethanethiol (gray curve; reaction time of $10 \mathrm{~min}$ ). The upper left inset shows an op-

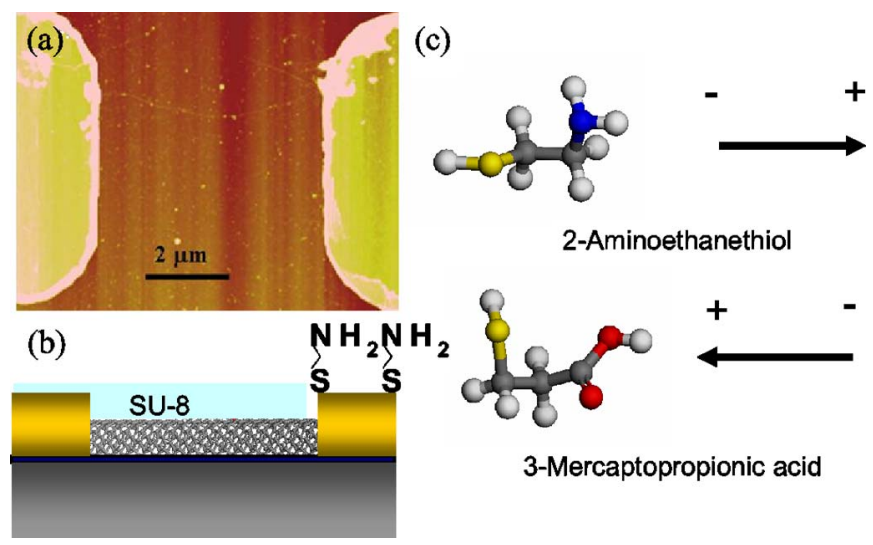

FIG. 1. (Color online) (a) AFM image of the device used in the experiment. (b) Schematic drawing of the SWNT-FET with a SAM on one contact. (c) Molecular structure of the two self-assembling molecules used in this study. 
(a)

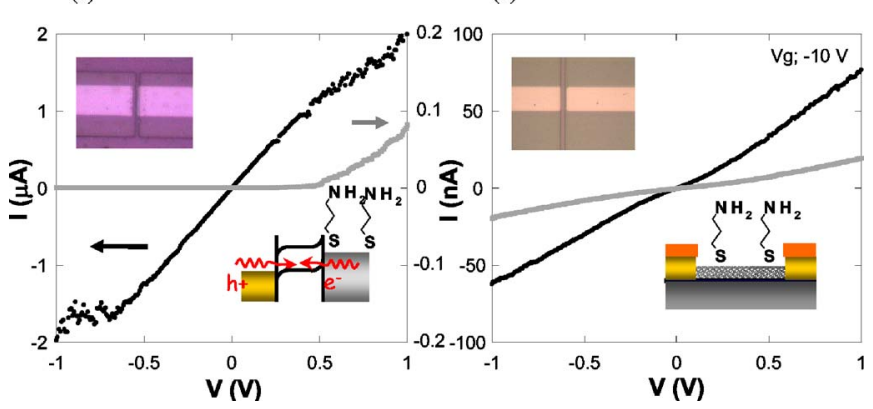

FIG. 2. (Color online) (a) $I-V$ curves measured before (black, at $V_{g}=0$ ) and after treatment of the surface with 2-aminoethanethiol (gray curve). Upper inset: optical microscope image of the single-contact opened device. Lower inset: schematic band diagram of the device. (b) $I-V$ curves before (black) and after (gray) 2-aminoethanethiol treatment in the contact-passivated device.

tical microscope image of the device, and the lower right inset shows the band diagram after the 2-aminoethanethiol treatment. As shown in Fig. 2(a), a symmetric $I$ - $V$ curve is observed before introduction of the SAM. After the 2-aminoethanethiol treatment, by contrast, the device exhibits a highly asymmetric, diodelike $I-V$ characteristic.

For comparison, we fabricated devices in which both contact electrodes were covered with SU-8, exposing only the nanotube body region [Fig. 2(b)]. After treatment of these SU-8-covered electrodes with 2-aminoethanethiol, the device showed symmetric $I-V$ characteristics with decreased conductance, as was previously observed by Kong and Dai, who attributed the conductance decrease to the electron doping nature of the amine groups. ${ }^{18}$ When we used a 3-mercaptopropionic acid SAM in similar experiments, no significant change of transport characteristics was observed (data not shown).

The diodelike operation of the SWNT-FET with a 2-aminoethanethiol SAM can be explained by the formation of asymmetric contacts after the molecular treatment of the cathode surface, as depicted in the inset of Fig. 2(a). A similar self-assembly approach has been used to control the Schottky barrier height in organic electronic devices. ${ }^{19,20} \mathrm{We}$ used two molecules, 2-aminoethanethiol and 3-mercaptopropionic acid, which are predicted to have different dipole directions [see Fig. 1(c)]. We found that of these two molecules, only treatment with 2 -aminoethanethiol resulted in diodelike operation.

Using $a b$ initio electronic structure calculations, we investigated the different effects of the 2-aminoethanethiol and 3-mercaptopropionic acid molecules on a bare Au surface. We used the same calculation method as in our previous work. $^{21}$ Here we adopted slab geometry with six gold layers, in which the two layers in the center were fixed during the geometry optimization calculation. Plotting the local potential with respect to the Fermi level, we obtain the work function of the bare $\mathrm{Au}$ surface as well as the 2-aminoethanethiol- and 3-mercaptopropionic-adsorbed $\mathrm{Au}$ surfaces, as shown in Figs. 3(a)-3(c), respectively. The calculated work function of the bare $\mathrm{Au}$ surface $(\approx 5.2 \mathrm{eV})$ is very close to the previously reported value. ${ }^{22}$ The work functions of the 2-aminoethanethiol- and 3-mercaptopropionicadsorbed Au surfaces [Figs. 3(b) and 3(c)] exhibit obvious differences. Specifically, adsorption of 2-aminoethanethiol moiecules substantially decreases the work function

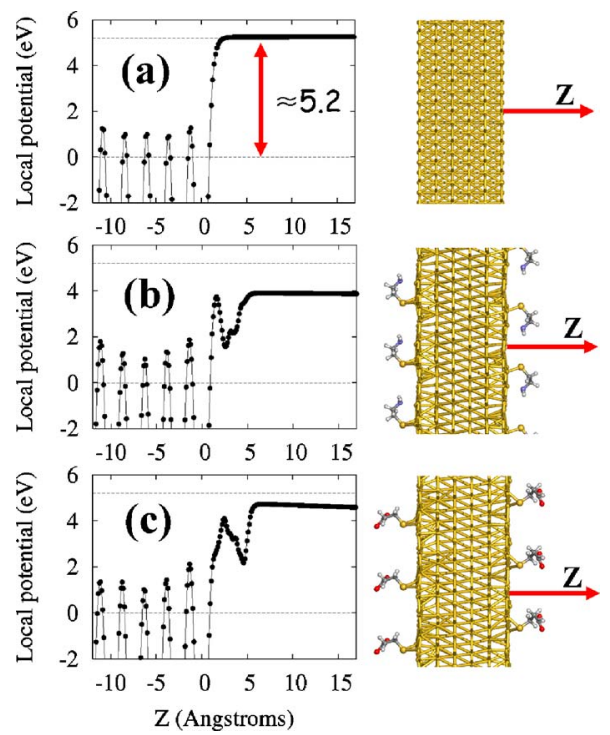

FIG. 3. (Color online) Plots of the local potentials with respect to the Fermi level of (a) the bare six layers of $\mathrm{Au}$, (b) the six layers of Au with adsorbed 2-aminoethanethiol molecules, and (c) the six Au layers with adsorbed 3-mercaptopropionic acid molecules.

$(\approx 3.9 \mathrm{eV})$, whereas adsorption of 3-mercaptopropionic acid has little effect $(\approx 4.7 \mathrm{eV})$. This difference in electronic structure confirms that the asymmetric $I-V$ pattern observed after treatment of one electrode of the SWNT-FET with 2-aminoethanethiol was due to an adjustment of the Schottky barrier at the treated contact.

To investigate the effect of treatment with 2-aminoethanethiol on the transfer character, we measured the evolution of the $I-V_{g}$ characteristics of the SWNT-FETs, as shown in Fig. 4(a). Before the reaction with 2-aminoethanethiol, the device shows typical $p$-type transistor behavior (black circles). After treatment with 2-aminoethanethiol for $10 \mathrm{~min}$ (empty squares) and $5 \mathrm{~h}$ (gray symbols), however, the $p$-channel conduction decreases and $n$-type conduction becomes dominant, as indicated by the solid arrows in Fig. 4(a). The effect of the 2-aminoethanethiol SAM seems to saturate by a reaction time of about $5 \mathrm{~h}$, with the $I-V_{g}$ characteristics remaining (a)

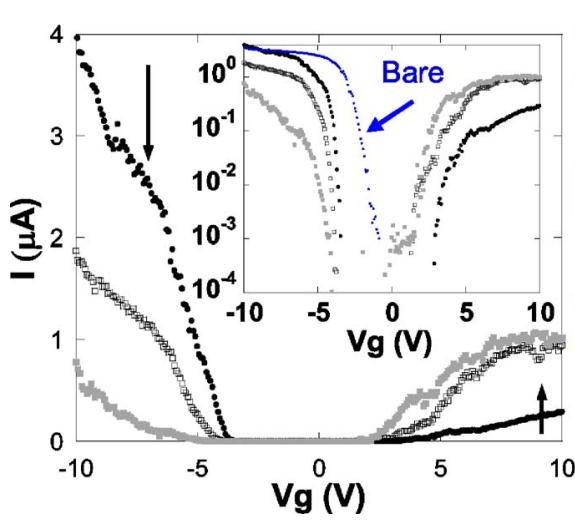

(b)

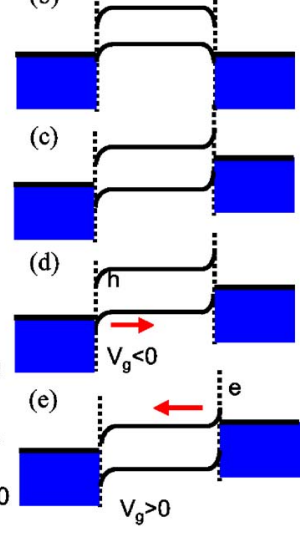

FIG. 4. (Color online) (a) Evolution of electrical transfer characteristics with the formation of a 2-aminoethanethiol SAM: before (black circles), after $10 \mathrm{~min}$ of reaction (empty squares), and after $5 \mathrm{~h}$ of reaction (gray squares). Inset shows a log-scale plot. Band diagram for the SWNT-FET (b) before and (c) after the SAM treatment The band diagram for the SWNTFET with one contact modified under (d) $V_{g}<0$ and (e) $V_{g}>0$. 
almost unchanged upon further treatment. As a result, the SWNT-FET with a 2-aminoethanethiol SAM on one contact shows an ambipolar transfer character. The ambipolar transfer character can be explained by considering the band diagram with an asymmetric contact, generated by SAM treatment of one contact. Before the SAM treatment [Fig. 4(b)], the Fermi levels of the electrodes are aligned with the valence band of the carbon nanotube, leading to $p$-type behavior. After the formation of the 2-aminoethanethiol SAM [Fig. 4(c)], however, the Fermi level of the cathode is aligned with the conduction band edge. For the SWNT-FET with a treated electrode the hole current would still dominate under the condition of $V_{g}<0$, as shown in Fig. 4(d). However, significant electron conduction will occur in the treated device when $V_{g}>0$, as shown in Fig. 4(e). As a result, the device shows ambipolar transfer character.

It is also noteworthy that the threshold voltage of the device does not change significantly after SAM treatment of one of the contact electrodes, as shown in Fig. 4(a). It is well known that shifts in the threshold voltage are largely due to variations in the doping level in the semiconductor channel region, whereas changes in the slope of $I-V_{g}$ curves derive from variations in the Schottky barrier at the contact. ${ }^{23}$ These findings also confirm that introduction of the SAM of 2-aminoethanethiol modifies the Schottky barrier at the engineered contact.

In summary, we have fabricated $p$-type SWNT-FETs and then converted them into diodes by tuning the Schottky barrier height using a SAM technique. Highly asymmetric, diodelike $I-V$ curves were observed when one of the contacts was treated with a 2-aminoethanethiol SAM. A $b$ initio electronic structure calculations confirmed that adsorption of 2-aminoethanethiol onto a $\mathrm{Au}$ surface induces a strong decrease in the work function, whereas adsorption of 3-mercaptopropionic acid has little effect. The present results suggest that tuning the Schottky barrier by introducing a SAM of a selected molecular species could be a practical method to control conduction patterns of nanotube-based electronic devices.
This work was supported by the MOST, the MOCIE (No. RTI 04-03-06), and the electron Spin Science Center at POSTECH, Korea. One of the authors (N.P.) would like to acknowledge the support from KISTI under the "7th Strategic Supercomputing Applications Support Program."

${ }^{1}$ R. Saito, G. Dresselhaus, and M. S. Dresselhaus, Physical Properties of Carbon Nanotubes (Imperial College, London, 1998).

${ }^{2}$ S. J. Tans, A. R. M. Verschueren, and C. Dekker, Nature (London) 393, 49 (1998).

${ }^{3}$ A. Javey, J. Guo, Q. Wang, M. Lundstrom, and H. Dai, Nature (London) 424, 6949 (2003).

${ }^{4}$ A. Javey, J. Guo, D. B. Farmer, Q. Wang, D. W. Wang, R. G. Gordon, M. Lundstrom, and H. Dai, Nano Lett. 4, 447 (2004).

${ }^{5}$ C. Zhou, J. Kong, E. Yenilmez, and H. Dai, Science 290, 1552 (2002).

${ }^{6}$ R. D. Antonov and A. T. Johnson, Phys. Rev. Lett. 16, 3274 (1999).

${ }^{7}$ J. U. Lee, Appl. Phys. Lett. 87, 073101 (2005).

${ }^{8}$ M. Freitag, M. Radosavljevic, Y. Zhou, and A. T. Johnson, Appl. Phys. Lett. 79, 3326 (2001)

${ }^{9}$ S. Heinze, J. Tersoff, R. Martel, V. Derycke, J. Appenzeller, and Ph. Avouris, Phys. Rev. Lett. 89, 106801 (2002).

${ }^{10}$ X. Cui, M. Freitag, R. Martel, L. Brus, and Ph. Avouris, Nano Lett. 3, 783 (2003).

${ }^{11}$ Y. Nosho, Y. Ohno, S. Kishimoto, and T. Mizutani, Appl. Phys. Lett. 86, 073105 (2005).

${ }^{12}$ H. Oh, J.-J. Kim, W. Song, S. Moon, N. Kim, J. Kim and N. Park, Appl. Phys. Lett. 88, 103503 (2006).

${ }^{13}$ C. Lu, L. An, Q. Fu, H. Zhang, J. Murduck, and J. Liu, Appl. Phys. Lett. 88, 133501 (2006).

${ }^{14}$ H. M. Manohara, E. W. Wong, E. Schlecht, B. D. Hunt, and P. H. Siegel, Nano Lett. 5, 1469 (2005).

${ }^{15}$ M. H. Yang, K. B. K. Teo, W. I. Milne, and D. G. Hasko, Appl. Phys. Lett. 87, 253116 (2005).

${ }^{16}$ J. Kong, H. T. Soh, A. M. Cassell, C. F. Quate, and H. Dai, Nature (London) 395, 878 (1998).

${ }^{17}$ A. Ulman, An Introduction to Ultrathin Organic Films; From LangmuirBlodgett to Self-assembly (Academic, San Diego, CA, 1991).

${ }^{18}$ J. Kong and H. Dai, J. Phys. Chem. B 105, 2890 (2001).

${ }^{19}$ B. de Boer, A. Hadipour, M. M. Mandoc, T. can Woudenbergh, and P. W. M. Blom, Adv. Mater. (Weinheim, Ger.) 17, 621 (2005).

${ }^{20}$ I. H. Campbell, S. Rubin, T. A. Zawodzinski, J. D. Kress, R. L. Martin, N. N. Barashkov, and J. P. Ferraris, Phys. Rev. B 54, 14321 (1996).

${ }^{21}$ N. Park and S. Hong, Phys. Rev. B 72, 045408 (2005).

${ }^{22}$ H. B. Michaelson, J. Appl. Phys. 77, 4729 (1977).

${ }^{23}$ V. Derycke, R. Martel, J. Appenzeller, and Ph. Avouris, Appl. Phys. Lett. 80, 2773 (2002) 